\title{
El aprendizaje a saltos mediante el uso de equipos de radio definida por software para la docencia en el grado en ingeniería electrónica de telecomunicación ${ }^{*}$
}

\author{
Joaquin Pérez Soler ${ }^{1}$, Adrián Suárez Zapata ${ }^{1}$, José Torres País ${ }^{1}$, Raimundo \\ García-Olcina $^{1}$, Julio Martos Torres ${ }^{1}$, Jesus Soret Medel ${ }^{1}$, Pedro A. Martínez \\ Delgado $^{1}$, Abraham Menéndez Márquez ${ }^{1}$ y David Garcia-Acosta ${ }^{2}$ \\ ${ }^{1}$ Departament d'Enginyeria Electrònica, Escola Superior Tècnica d'Enginyeria, Universitat de \\ València \\ ${ }^{2}$ Departament d'Enginyeria Informática, Escola Superior Tècnica d'Enginyeria, Universitat de \\ València
}

\begin{abstract}
How to design communications systems and its foundations depends on various factors such as the accessibility to learning resources, the academic environment and the audience itself. This article reports how the introduction of learning methodologies based on project based learning (PBL), practical devices and asynchronous working has modified the perception of the students about their learning aptitudes. The combination of traditional and offline sessions, with the PBL methodology to perform practical communications systems demonstrations based on professional SDR devices by group of students is presented and analysed in this article. The quantified outcome of their academic involvement due to this innovation is evaluated. The indicators shows that the overall dimensions of student learning of communications systems were improved compared with previous sessions based on simulated, non-groupal and magistral lectures.
\end{abstract}

Keywords: communications, SDR, PBL, peer review, hybrid teaching, asynchronous learning

\begin{abstract}
Resumen
La enseñanza de las comunicaciones conlleva situaciones de aprendizaje diferentes según el contexto en el que se desarrollen. El contexto actual conlleva el cambio de modelo del habitual totalmente presencial en la docencia a situaciones hibridas en línea y presenciales. Esta situación plantea retos como el desarrollar el aspecto práctico del
\end{abstract}

\footnotetext{
*Este trabajo ha sido financiado por el "Vicerectorat d'Ocupació i Programes Formatius" de la Universitat de València a través del proyecto UV-SFPIE_PID20-1354055.
} 
El aprendizaje a saltos mediante el uso de equipos de radio definida por software para la docencia en el grado en ingeniería electrónica de telecomunicación

aprendizaje de los contenidos de comunicaciones y en segundo lugar armonizar las limitaciones de presencialidad con aplicaciones prácticas de las mismas. Este proyecto de innovación docente pretende desarrollar metodologías de trabajo cercanos a los entornos profesionales en el ámbito de la Ingeniería mediante el uso de dispositivos específicos como son los basados en radio definidas por software. Estos dispositivos conjuntamente con una metodología de aprendizaje basada en proyectos (ABP) y una enseñanza que combine la docencia asíncrona y presencial, a saltos, permitirá crear un entorno muy favorable para la adquisición de competencias y habilidades profesionales de forma práctica. La puesta en marcha de sesiones híbridas para el desarrollo de proyectos de sistemas de telecomunicaciones con docencia asincrona y trabajo en grupo se ha evaluado en este artículo. El grupo bajo estudio se ha evaluado mediante encuestas de tipo likert y ha mostrado una mejora en todos los aspectos evaluados superiores al $40 \%$ frente a la situación inicial sin estas innovaciones.

Keywords: telecomunicaciones, radio definida por software, ABP, docencia híbrida, revisión por pares, aprendizaje asíncrono

\section{Introducción}

Esta investigación y sus resultados presentados en este artículo se enmarcan en la línea de innovación docente sobre las necesidades de formación en competencias e innovación en el aula aplicando las metodologías STEM (Ciencia, Tecnología, Ingeniería y Matemáticas) como estrategia pedagógica. Partiendo de las experiencias del equipo de trabajo en PID de cursos anteriores en la Escola Tècnica Superior d'Enginyeria de la Universitat de València en materias STEM como la robótica, se pretende desarrollar y aplicar nuevas estrategias docentes en la impartición de materias relacionadas con los sistemas de telecomunicación en el área de tecnología electrónica en el grado en Ingeniería Electrónica de Telecomunicación (GIET) en la Universitat de Valencia (UV).

Se toma como objetivo desplazar el foco de los contenidos expositivos a la aplicación práctica, motivadora y transversal de los conocimientos en el aula de laboratorio. En una primera implementación se ha tomado como objetivo inicial de investigación la asignatura de tipo troncal Sistemas y Servicios de Telecomunicaciones (SST), emplazada en el $3^{\underline{0}}$ curso GIET en primer cuatrimestre. Esta asignatura se imparte en l'Escola Tècnica Superior d'Enginyeria (ETSE) de la Universitat de València.

En este proyecto se busca variar la metodología de aprendizaje actual de estas sesiones prácticas hacia una basada en proyectos que permita combinar el aprendizaje presencial y el asíncrono. Este trabajo de innovación docente busca optimizar la presencialidad del alumnado y, a su vez, permitir la adquisición de habilidades profesionales del egresado, mediante trabajo en equipo, estrategias de aprendizaje activo y uso de plataformas y equipos profesionales de trabajo.

Por ello el proyecto incide en la necesidad de introducir en la metodología de enseñanza las plataformas de comunicaciones profesionales como son las basadas en radio definidas por software (SDR). Estas plataformas SDR (Mitola 1993; Jiménez y col. 2017) usan dispositivos que digitalizan la señal de radio frecuencia y envían las muestras a un ordenador, donde se procesan para extraer información. Estos dispositivos han revolucionado el aprendizaje en telecomunicaciones puesto que implementan una gran mayoría de los sistemas mediante programas informáticos configurables en tiempo real (Botella y col. 2020; Stewart y col. 2015). En estos casos un solo dispositivo SDR puede permitir configurar su uso con estándares como el DVB-T, WiFi (IEEE 802.11), Bluetooth 
o LTE (4G). A su vez estos estándares de comunicaciones son parte del contenido docente de la asignatura bajo estudio.

En el contexto actual en la Universitat de València se busca conjugar el aprendizaje activo con la salud individual del alumnado y por ello se ha optado este curso académico 2020-2021 por un modelo híbrido de enseñanza. Pero en las prácticas es necesario enfatizar la parte presencial y experimental de los contenidos. Para poder atender las situaciones del alumnado derivadas de este contexto se opta por introducir equipos profesionales de comunicaciones de radio definida por software (SDR) conjuntamente con programas de procesamiento matemático. Esto permite tener un contexto de aprendizaje tanto presencial como virtual que posibilite trabajar al alumnado habilidades transversales del grado como son liderazgo, trabajo en grupo, presentación y hablar en público y de tipo profesional como son análisis y resolución de problemas, diseño de sistemas de comunicaciones entre otros. Por ello como se indica en la literatura el uso de Aprendizaje basado en proyectos (ABP) es crucial para la adquisición de competencias en el área de la Ingeniería (Chen, Kolmos y Du 2021; Sanger y Ziyatdinova 2014) .

Por ello este articulo busca evaluar como el uso de la plataforma SDR y el software de procesado matemático MATLAB (disponible para toda la comunidad universitaria de la UV) puede incidir en mejoras en el aprendizaje de los sistemas de comunicaciones en su vertiente práctica. Ante la situación de emergencia sanitaria actual, esto conllevará una racionalización de la ocupación de laboratorios de la ETSE-UV al permitir saltos de aprendizaje (HOP-LEARNING) entre presencial y asíncrono durante el desarrollo del proyecto, adaptándose al ritmo del alumnado.

El trabajo aquí presentado se estructura a continuación de la siguiente manera. En la sección 2 se definen los objetivos del proyecto de innovación docente relativos a este estudio en la sección 2 . La sección 3 analiza el desarrollo, las metodologías y acciones relevantes que se han implementado en este estudio. Finalmente las sección 4 presenta los resultados obtenidos y un análisis de los mismos. Se finaliza en la sección 5 con las conclusiones más relevantes del estudio presentado.

\section{Objetivos}

Los objetivos de la acción innovadora docente analizada en esta publicación se centran en la mejora de la calidad docente desde la perspectiva del alumnado aplicando metodologías de docencia asíncrona y síncrona, participativa y cercana al mundo profesional. Para ello se han planificado el uso de elementos SDR en prácticas de laboratorio, sesiones asíncronas para el desarrollo y guía de las sesiones y prácticas de revisión por pares de los resultados obtenidos.

En concreto se pueden observar los siguientes objetivos generales en esta investigación:

- Introducir los conceptos de aprendizaje basado en proyectos para el estudio y diseño de sistemas de comunicaciones.

- Adaptar la evaluación y la docencia práctica de los laboratorios mediante el uso de la plataforma SDR.

- Preparar y adaptar los materiales adecuados para la nueva situación docente.

- Reforzar las competencias asociadas al desarrollo de sistemas de telecomunicaciones actuales mediante el uso de una plataforma SDR y un software, ambos reconocidos en los ámbitos profesionales que se pueden encontrar los egresados en su futuro entorno laboral. 
El aprendizaje a saltos mediante el uso de equipos de radio definida por software para la docencia en el grado en ingeniería electrónica de telecomunicación

- Impulsar el aprendizaje colaborativo, liderazgo, resolución de problemas y trabajo en entornos laborales asíncronos, entre otras competencias formativas del alumnado del grado mediante una enseñanza a saltos (HOP-LEARNING).

- Evaluar el impacto en el aprendizaje del alumnado tras la incorporación de las innovaciones docentes.

Para la consecución de estos objetivos se ha determinado que los estudiantes de tercer curso del grado en Ingeniería Electrónica de Telecomunicación dispongan de plataformas SDR y material previo elaborado por profesionales para desarrollar las diferentes demostraciones. Los estudiantes dispondrán de sesiones presenciales síncronas y asíncronas y apoyo virtual para la realización del proyecto propuesto.

Finalmente, para poder cuantificar la innovación docente realizada se han evaluado las dimensiones de la motivación del aprendizaje e implicación del alumnado en 4 niveles: energía, absorción, satisfacción y dedicación, adaptando la propuesta de (Botella y col. 2020).

\section{Desarrollo de la innovación}

Para analizar la consecución de estos objetivos se han propuesto acciones en la parte práctica de laboratorio dentro de la asignatura bajo estudio. Esta parte de laboratorio está programada en base a sesiones independientes que tratan una temática de la guía docente, mediante el desarrollo guiado de un escenario práctico. Como denominador común en todas ellas es la no interacción con dispositivos reales de comunicaciones que están presentes en los actuales servicios y sistemas de comunicaciones. Tienen una fuerte carga abstracta y de desarrollo cognitivo mediante clase maestra y trabajo en grupo. Para ello se utilizan herramientas de simulación de sistemas, de tipo óptico como Optiwave (C) y de procesado de señales y datos como Matlab (C) .

La asignatura bajo estudio SST se ha impartido durante el primer cuatrimestre del curso académico 2020-2021. SST tiene matriculados 45 personas, de las cuales asisten de forma activa a las sesiones de laboratorio 39 de ellos. En las sesiones previas de prácticas se organizan en parejas para la realización de los contenidos planteados y se evalúa su desarrollo mediante entrega de memorias breves y realización de test al finalizar la sesión práctica.

En este contexto, la innovación docente planteada opta por introducir metodologías activas de aprendizaje mediante el desarrollo de un proyecto de forma grupal y mediante la evaluación final del mismo por pares. En los proyectos se opta por el uso de dispositivos de radio definida por software (SDR) para la realización de un sistema/servicio de telecomunicación. El trabajo se organiza en grupos de tamaño medio (2-5 estudiantes) y se enmarca temporalmente en las dos últimas sesiones programadas, que inicialmente estaban dedicadas a sistemas de comunicaciones móviles y a una sesión de evaluación de los conocimientos de laboratorio. Se disponen de 15 días naturales entre una sesión y la otra, lo que permite una organización temporal más abierta y adaptada a las necesidades de cada grupo. En la última sesión los grupos deben presentar/demostrar sus proyectos y sus pares deben evaluar su trabajo mediante una rúbrica. Para finalizar el proyecto cada grupo debe diseñar un guión del mismo contenido que los de laboratorio con preguntas clave, para que sirva de guía para otros alumnos en otros cursos del mismo área de conocimiento, como Fundamentos de las Comunicaciones ( $2^{\mathrm{O}}$ curso de GIET). 
El alumnado dispone de un guía de trabajo sobre como realizar estos proyectos, de la rúbrica de evaluación de los mismos, y de ítems de autoevaluación intermedia para analizar su progreso. A su vez se les presenta ejemplos de proyectos iniciales a modelar con estos dispositivos, usando el repositorio abierto de Matlab Central para uso de sistemas SDR.

\subsection{Organización y contenido de las sesiones bajo estudio}

El desarrollo de esta innovación se organiza en dos sesiones síncronas híbridas de laboratorio y otra sesión de uso libre.

La primera sesión es síncrona en directo en el aula, el alumnado asiste durante el horario de la sesión tanto en el aula como en remoto. Se presenta el tipo de dispositivos SDR y sus usos en diseño de sistemas y servicios de comunicaciones. Se graba y deja disponible en aula virtual de la UV la sesión realizada, aula virtual es un espacio virtual de docencia privado de la asignatura para todo alumnado matriculado. Se acorta la duración de la sesión magistral de 3 horas a 1 hora, y se dejan 2 horas de trabajo autónomo en grupos pequeños, en espacios dispuestos entre el aula de laboratorio, el aula de refuerzo y el espacio virtual de reunión $M S T E A M S$ para cumplir con las normas sanitarias COVID19. En esta sesión muchos alumnos (25\%) siguieron y trabajaron con su grupo de forma virtual. A su vez disponen de materiales previos de demostraciones de SDR mediante Matlab para desarrollar sus proyectos. En la primera sesión Se formaron 6 y 7 grupos de trabajo en cada uno de los laboratorios de la asignatura.

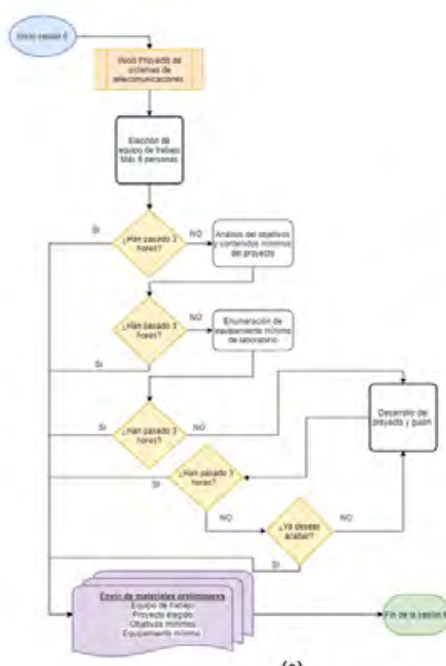

(a)

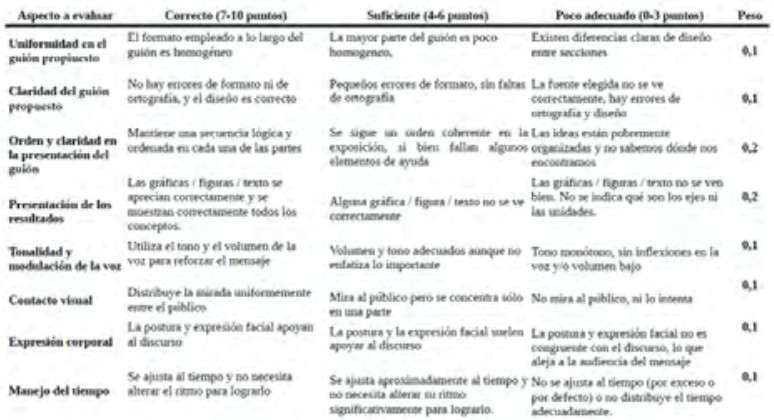

(b)

Fig. 1: Diagrama de trabajo de la sesión inicial y rubrica de las demostraciones finales.

Para poder seguir el desarrollo de la innovación docente los grupos de trabajo disponen de un esquema visual en la primera sesión, ver Figura 1 a), de los pasos iniciales necesarios para que el proyecto sea evaluado correctamente. En esta sesión se les pide que completen unas preguntas relativas al proyecto, trabajo elegido y condiciones de trabajo en grupo. Estas se deben entregar en una tarea de aula virtual antes de finalizar el horario de la sesión práctica. El objetivo es que 
El aprendizaje a saltos mediante el uso de equipos de radio definida por software para la docencia en el grado en ingeniería electrónica de telecomunicación

analicen su propio plan de trabajo y motivarles a tomar decisiones al respecto para el desarrollo del proyecto adecuadamente. Este paso previo se corresponde con la etapa de diseño y análisis previo de las condiciones de un sistema o servicio de comunicaciones.

La segunda parte de este proyecto se enmarca temporalmente entre la primera y siguiente sesión (14 días naturales). Esta segunda parte consiste en motivación para el aprendizaje propio y voluntario. Se dispone para el alumnado de horarios de laboratorio abierto que pueden o no utilizar. Deben reservarlo con mínimo de 12 horas de antelación e indicar los materiales que van a utilizar. Es una acción voluntaria por parte de cada grupo y conlleva una reflexión sobre el estado del proyecto y las condiciones para su ejecución/demostración en laboratorio. Al ser voluntario y de aula abierta es totalmente asíncrona y libre esta actividad de aprendizaje respecto al programa de la asignatura. En este caso 8 grupos de 13 totales usaron los espacios y tiempos de trabajo autónomo disponible. cabe destacar que todos los grupos dispusieron desde la sesión 1 de material específico de trabajo como dispositivos SDR receptores como RTL-SDR y transceptores SDR más complejos como ADALMPLUTO. Y también de materiales de conexiado RF como cables SMA, USB y de software de procesado con paquetes integrados como MATLAB y Simulink.

Tras esta etapa de trabajo asíncrono y libre se programa la sesión final, 14 días después de la inicial. Esta sesión de 3 horas de duración se organiza de la manera siguiente, 90 minutos de trabajo en grupo, 10 minutos de descanso y preparación del demostrador, y 80 minutos para las presentaciones/demostraciones de los proyectos y su evaluación. En la Figura 2 se pueden observar capturas de ejemplos de demostradores realizados durante las sesiones. La evaluación se realiza mediante rúbrica, ver ejemplo en Figura 1 b), disponible desde la sesión inicial. En esta sesión final nuevamente dada la situación sanitaria actual cerca del $20 \%$ del alumnado se conecto y participó de forma remota.

La evaluación final de todo el proyecto tiene en cuenta la nota obtenida en la rúbrica por los pares $(60 \%)$ y el profesor $(20 \%)$, la entrega de los resultados iniciales $(5 \%)$ y de una memoria final $(15 \%)$. Para la entrega de la memoria final se indica que no debe ser exhaustiva sino explicativa del proceso de desarrollo de su proyecto. Se les entrega un guión de contenidos mínimos a entregar en la memoria final.
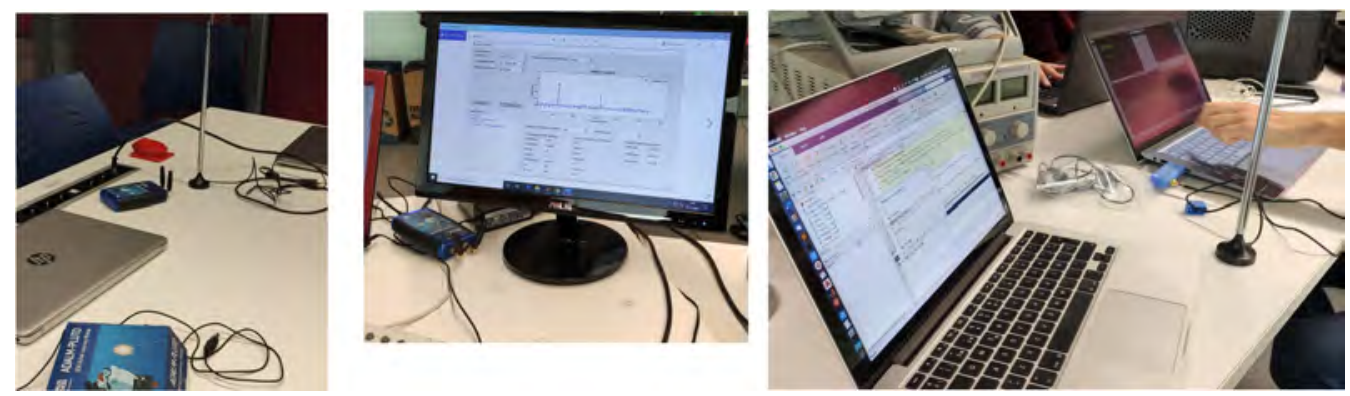

Fig. 2: Capturas de ejemplos de los proyectos y demostradores SDR desarrollados.

Con este planteamiento se evalúa el trabajo en grupo, las habilidades de laboratorio mediante uso de dispositivos SDR, el desarrollo de un proyecto de comunicaciones en todas sus etapas y su capacidad de evaluar y analizar el trabajo de otros grupos. Cabe destacar que esta estructuración permite desarrollar y alcanzar los objetivos iniciales de impulsar el aprendizaje colaborativo, li- 
derazgo, resolución de problemas y trabajo en entornos laborales asíncronos. En concreto, como indican estudios previos este tipo de metodología aprendizaje-servicio ABP es muy adecuada para desarrollar competencias transversales como el trabajo autónomo, la capacidad de organización y planificación, trabajo colaborativo, toma de decisiones o razonamiento critico analítico (Perrenet, Bouhuijs y Smits 2000; Chen, Kolmos y Du 2021). En este caso se ha implementado mediante un planteamiento de aprendizaje a saltos, donde se parte de la clase magistral síncrona en remoto y en directo, para tener aprendizaje autónomo asíncrono y finalmente sesión de evaluación síncrona. Y permite la introducción y uso de dispositivos de comunicaciones SDR que se contraponen al modelo magistral de sesiones previas.

También es reseñable como la temporalización propuesta que es propia de ABP ha permitido trabajar los aspectos de trabajo en grupo y toma de decisiones. En particular, las tareas planteadas conllevan toma de decisiones sobre el proyecto, escoger grupo de trabajo, interesarse por acudir a sesiones de acceso libre para desarrollar su trabajo autónomo y otras acciones descritas previamente han tenido un éxito importante (más del $70 \%$ de los alumnos usaron las sesiones de acceso libre). Estas acciones permiten desarrollar y alcanzar los objetivos propuestos en términos de trabajo y desarrollo de competencias formativas y transversales.

Para poder evaluar el impacto de esta innovación docente de introducción de dispositivos SDR, aprendizaje a saltos y metodologías activas de aprendizaje el alumnado realizado un test anónimo sobre su visión de la asignatura al inicio del curso de sesiones prácticas de la asignatura (final de octubre 2020) y el mismo test al finalizar la última sesión tras la presentación de los proyectos de comunicaciones (final de diciembre 2020).Este tipo de encuesta permite valorar de forma indirecta también la consecución de los objetivos ligados a competencias formativas transversales anteriormente descritas

\section{Resultados}

Para poder evaluar el impacto del uso de dispositivos SDR en el desarrollo de la enseñanza se ha utilizado un cuestionario para evaluar la motivación y compromiso del alumnado en las diferentes fases del mismo. Para ello se ha adaptado el cuestionario de (Schaufeli y Bakker 2004; SorianoAsensi y col. 2019; Botella y col. 2020). Este cuestionario es una herramienta de análisis objetivo que contempla dimensiones de la experiencia educativa del alumnado como son la energía, la absorción de conocimientos, la satisfacción y la dedicación (García-Ros y col. 2018; Schaufeli y Bakker 2004). En este caso el alumnado contestó una batería de 10 preguntas, valorando en una escala Likert de 1 (nunca) a 5 (casi siempre/siempre), de forma totalmente anónima.

Como indicaron trabajos anteriores (Botella y col. 2020) este cuestionario el objetivo es cuantificar tres dimensiones de la implicación académica: i) energía, relacionada con la capacidad de resiliencia del alumnado frente a la resolución de problemas (Q1, Q4, Q8); ii) absorción, relacionada con la capacidad del alumnado para concentrarse en aquellas tareas que está realizando (Q3, Q6, Q9); y iii) dedicación,relacionada con la percepción del alumnado sobre la relevancia de las actividades que realiza (Q2, Q5, Q7). La satisfacción general se evalúa mediante la pregunta Q10. A continuación se adjunta la encuesta utilizada durante las sesiones prácticas objetivo de la innovación. 

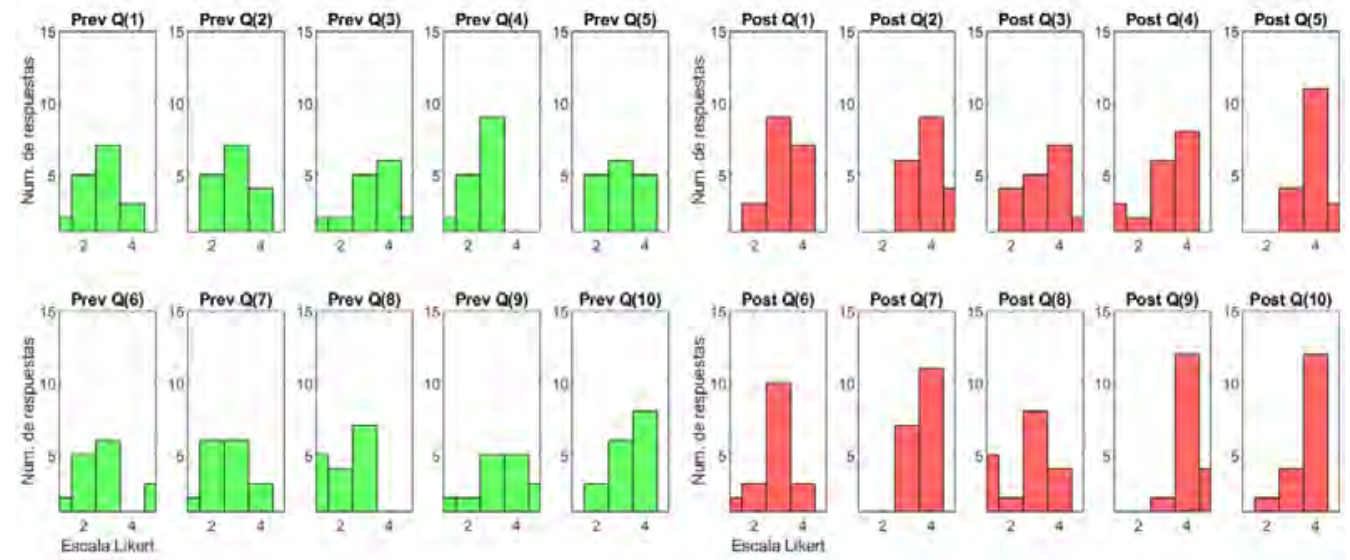

(a)

(b)

Fig. 3: Histograma de respuestas y valoraciones recibidas en cada pregunta.

La siguiente encuesta se realiza dentro del marco de un proyecto de innovación docente financiado para el curso 2020-2021 por la Universitat de València. Las respuestas son anónimas, y buscan mejorar la docencia en el área de telecomunicaciones del grado GIET.

Destaca hasta qué punto las afirmaciones siguientes describen tu situación en ESTE ÚLTIMO MES con respecto a las prácticas de SST y la asignatura. Responde con sinceridad, utilizando la siguiente escala entre 1 (nunca/casi nunca) y 5 (casi siempre/siempre)

- Q1 - En la universidad me siento lleno/llena de energía;

- Q2 - Pienso que las prácticas son relevantes y significativas;

- Q3 - El tiempo me parece que "vuela" cuando estoy estudiando;

- Q4 - Me siento con gran fuerza y vigor mientras estudio;

- Q5 - Me entusiasma lo que estudio;

- Q6 - Cuando estoy trabajando o estudiando me olvido de todas las cosas que pasan a mi alrededor;

- Q7 - El trabajo en la universidad me resulta ilusionante;

- Q8 - Tengo ganas de ir a clase cuando me levanto por la mañana;

- $\mathbf{Q 9}$ - Me satisface trabajar con intensidad en mi carrera;

- Q10 - En general, me siento muy satisfecho con mis estudios y el trabajo realizado en la universidad. 


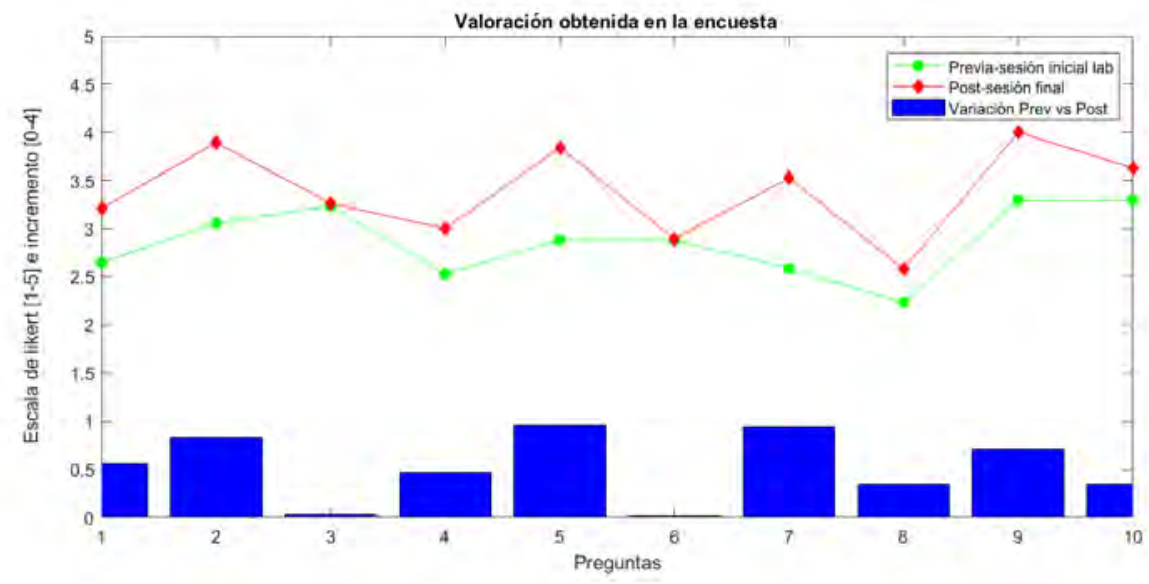

Fig. 4: Media de las respuestas recibidas y variación entre la sesión inicial y final.

En la Figura 3 se observa el histograma de las respuestas recibidas. En la primera encuesta realizada, Figura 3 a), tras la primera sesión práctica programada a inicios de noviembre 2020 se obtuvieron 17 respuestas completas. La encuesta final se realizó al acabar la sesión final de proyecto con SDR a finales de diciembre 2020 con 19 respuestas recibidas, resultados en Figura 3 b). Se observa como la mayoría de respuestas recibidas en las de tipo energía mejoran y se sitúan en el 4 tras comenzar con mayoría de 3. En cambio, las de tipo absorción no sufren grandes cambios, esto puede deberse al clima actual de incertidumbre que conlleva realizar los estudios en las condiciones de salud y socialización actuales. Este contexto social no tuvo grandes cambios durante ese periodo de 9 semanas entre octubre-noviembre-diciembre 2020. Sin embargo la dedicación a la asignatura si se ha visto incrementada tras la innovación docente y esto se ha reflejado en un aumento de la satisfacción general como se observa en la Figura 4.

De forma desagregada, a los resultados muestran que inicialmente la implicación del alumnado era baja o media y esto no es meramente responsabilidad de la materia si no como indicas otros estudios, es propio del contexto COVID actual (Balluerka Lasa y col. 2020). Se observa claramente que el mero hecho de cambio de tipo de sesiones más prácticas con mayor libertad de decisión y empoderamiento de los estudiantes no es por si solo un hecho diferenciador en su implicación académica, ver Q3 y Q6. Sin embargo, la posibilidad de planificar el diseño de la tarea y poder demostrarla al resto de compañeros supone un gran cambio en la percepción y absorción de la materia, como se observa en la Q9 con mas de 0,7 puntos de cambio. La introducción del método de aprendizaje basado en problemas junto con la posibilidad de uso de herramientas profesionales de telecomunicaciones, acercando demostraciones reales al laboratorio, como un receptor de señales radio de aeronaves ADS-B, se ha reflejado en la dimensión energía (Q2,Q5 y Q7) de forma muy clara con aumentos entre 0,5 y 0,9 puntos frente a la situación inicial. Cabe destacar que en los proyectos entregados muchos grupos implicaron una motivación personal y profesional en el mismo, por ejemplo contextualizando el uso de ADS-B en aeronaves recreativas o en el diseño del receptor y la reglamentación en situaciones de seguridad nacional. Y esta implicación es la que se ve reflejada en la encuesta. El hecho de poder demostrar y obtener proyectos que funcionaran gracias a la versatilidad de los elementos SDR supone una recompensa al trabajo realizado por el alumnado. Esto se ha reflejado en obtener en esta sesión notas un $20 \%$ superiores en media a las conseguidas en las sesiones prácticas previas. 
El aprendizaje a saltos mediante el uso de equipos de radio definida por software para la docencia en el grado en ingeniería electrónica de telecomunicación

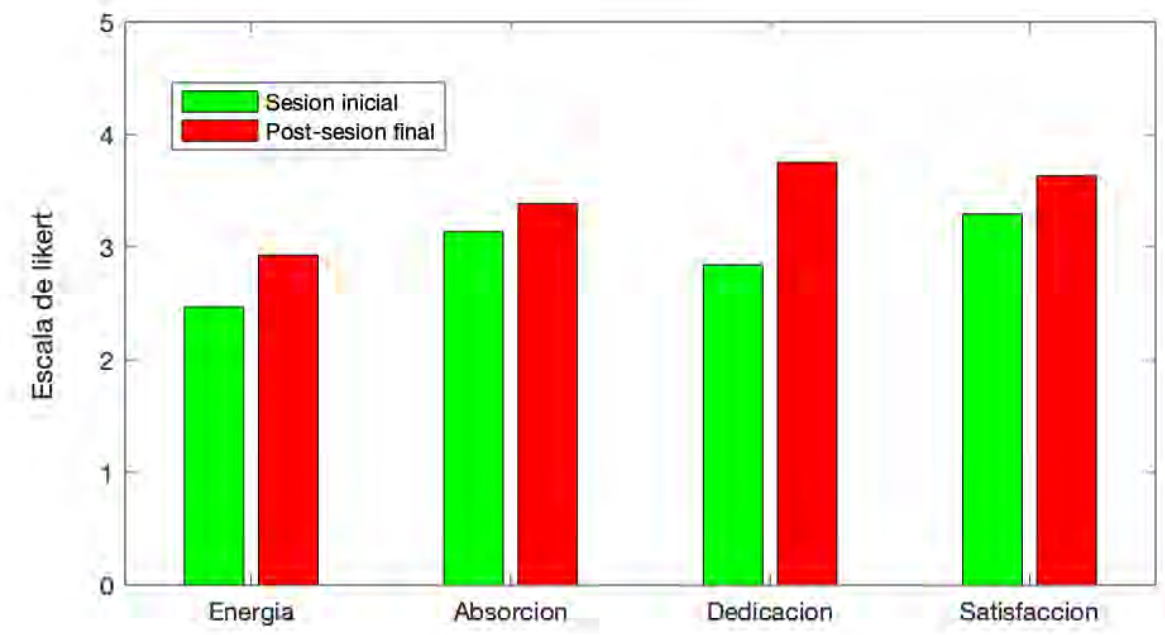

Fig. 5: Dimensiones evaluadas del test y variación entre sesión previa y posterior de laboratorio.

Si consideramos las 4 dimensiones de la motivación e implicación del alumnado en la Figura 5 se observa un incremento en todas ellas y especialmente en el aspecto de dedicación y energía. Sin embargo la concentración y absorción de los contenidos desarrollados no sufre una variación significativa, menor a 0.3. El contexto de absorción implica elementos de concentración, abstracción, realización y maestría que necesitarían de unas condiciones psicológicas y sociales más estables que las actuales. Y en lineas generales la satisfacción (Q10) ha mejorado cerca del 0.3 tras la introducción de las innovaciones docentes explicadas.

Los resultados muestran en primer lugar una participación alta en la innovación planteada por parte del alumnado, al alinear sus conocimientos previos con aplicaciones profesionales de comunicaciones y con sus motivaciones personales al cursar este grado GIET. Esto se muestra en las variaciones de la valoración de las cuestiones relacionadas con la energía, variación de 2.47 a 2.93 de valoración, y de dedicación, variación de 2,84 a 3,75 de valoración (0.9 puntos). Esto se ha reflejado también en los resultados obtenidos en cada uno de los apartados evaluables con una media de 9,4 puntos en esta sesión frente a los 7,5 puntos de las anteriores. También el uso de aprendizaje a saltos adaptado a las necesidades del alumnado, gracias al uso de herramientas de comunicaciones en tiempo real, y uso de componentes profesionales fuera del horario indicado en el plan de estudios ha supuesto una mejora en la percepción general de la asignatura con un aumento en el valor de satisfacción general de 3,26 a 3,64. Finalmente, la dimensión absorción como se ha comentado anteriormente es difícil de cambiar de forma radical con este tipo de acciones. Cabe indicar que estas dimensiones también permiten evaluar de forma indirecta la consecución de los objetivos planteados en cuanto a competencias transversales y formativas. El desarrollo de estas competencias como se ha descrito en la sección 3. El trabajo en grupo, el desarrollo de aprendizaje autónomo y la mejora en la capacidad expositiva y la implicación en empatizar con el trabajo de otras personas mediante su evaluación por pares actúan sobre elementos de valoración anteriores de forma indirecta como son la dedicación y la energía. Por ello aunque no exista una valoración directa si que se observa un traslado del trabajo exitoso de las competencias a las dimensiones evaluadas en esta innovación docente. El 
éxito que avalan estos resultados muestran la necesidad de actuar en esta línea de trabajo de forma más continuada para mejorar no los aspectos profesionales del egresado sino también el contexto en el cual deben desarrollarse. Puesto que esta innovación docente aquí planteada se ha realizado en el contexto de emergencia sanitaria actual y no debe olvidar que su objetivo es como universidad ofrecer una docencia de calidad que permita a los estudiantes adquirir las competencias de su titulación. Por ello como se comenta en (Sánchez-Carracedo y col. 2020) es necesario contar con herramientas en la educación universitaria desde la administración, docencia y alumnado que promuevan la calidad docente y se adapte al contexto en el cual se realicen. Este estudio muestra resultados satisfactorios en esta dirección.

\section{Conclusiones}

La introducción de elementos SDR, junto con la programación de docencia asíncrona y síncrona (a saltos), en un entorno de docencia híbrida y de aprendizaje basado en proyectos ha supuesto una mejora evidente en la implicación del alumnado de SST de GIET de la ETSE-UV en más de 0,3 puntos (sobre 5 en escala Likert) en todos los ámbitos cuantificables como son energía, absorción, dedicación y satisfacción general. A su vez ha supuesto un claro cambio de tendencia al aportar en primer lugar un entorno práctico de trabajo muy versátil y cercano a las actividades de comunicaciones de su perfil de profesional con el uso de dispositivos SDR. En esta comunicación se ha mostrado como los objetivos iniciales planteados por la innovación docente se han desarrollado y alcanzados en su totalidad. La introducción de una la línea de trabajo definida por un proyecto en grupo ha mejorado la percepción de la asignatura y ayudado a motivar el desarrollo de competencias transversales del grado, como son el trabajo en grupo, liderazgo, toma de decisiones, elaboración de informes y se refleja en las dimensiones de energía y dedicación con mejoras de valoración de 0,5 y 0,9 puntos respectivamente. Como se ha indicado anteriormente, el desarrollo de las competencias se ha evaluado de forma indirecta por estas dimensiones, para futuros trabajos se trabajará en evaluaciones más directas de las mismas en las valoraciones del estudiantado que busquen demostrar de forma directa su influencia. En concreto las preguntas 5 y 7 que llevan en su enunciado la ilusión y emoción por el trabajo desempeñado mejoran un 0,96 su valoración inicial, dato que sugiere que este tipo de acciones motivan y mejoran la percepción del alumnado frente a los contenidos académicos de comunicaciones. Por otro lado las preguntas Q3 y Q6 solo mejoran 0,01 puntos, en relación a como influye el contexto en el estudiantado, ante lo cual este aspecto de absorción debería ser revisado y puesto en valor en próximos estudios de innovación docente en el ámbito de las enseñanzas de comunicaciones en el grado en ingeniería electrónica de telecomunicación en la ETSE UV.

\section{Referencias bibliográficas}

Balluerka Lasa, Miren Nekane y col. (2020). Las consecuencias psicológicas de la COVID-19 y el confinamiento. Inf. téc. Servicio de Publicaciones de la Universidad del País Vasco.

Botella, Carmen y col. (sep. de 2020). "Evaluación del impacto del uso de dispositivos de radio definida por software como herramienta docente en la materia de comunicaciones digitales". En: XXXV Simposium Nacional de la Unión Científica Internacional de Radio. URSI, págs. 1-4. 
El aprendizaje a saltos mediante el uso de equipos de radio definida por software para la docencia en el grado en ingeniería electrónica de telecomunicación

Chen, Juebei, Anette Kolmos y Xiangyun Du (2021). "Forms of implementation and challenges of PBL in engineering education: a review of literature". En: European Journal of Engineering Education 46.1, págs. 90-115.

García-Ros, R. y col. (sep. de 2018). "The schoolwork engagement inventory: factorial structure, measurement invariance by gender and educational level, and convergent validity in secondary education (12-18) years." En: Journal of Psychoeducational Assessment 36.6, págs. 588-603.

Jiménez, Víctor P Gil y col. (2017). "Learning mobile communications standards through flexible software defined radio base stations". En: IEEE Communications Magazine 55.5, págs. 116-123.

Mitola, Joseph (1993). "Software radios: Survey, critical evaluation and future directions". En: IEEE Aerospace and Electronic Systems Magazine 8.4, págs. 25-36.

Perrenet, Jacob C, Peter AJ Bouhuijs y Jan GMM Smits (2000). "The suitability of problembased learning for engineering education: theory and practice". En: Teaching in higher education 5.3, págs. 345-358.

Sanger, Phillip A y Julia Ziyatdinova (2014). "Project based learning: Real world experiential projects creating the 21st century engineer". En: 2014 International Conference on Interactive Collaborative Learning (ICL). IEEE, págs. 541-544.

Schaufeli, W. y A. Bakker (dic. de 2004). UWES-Utrecht Work Engagement Scale: Test manual. Vol. 57. 11. Department of Psychology Utrecht University Utrecht The Netherlands., págs. 14-19.

Soriano-Asensi, Antonio y col. (2019). "Aprendizaje basado en proyectos en los laboratorios de comunicaciones digitales". En: IN-RED 2019. V Congreso de Innovación Educativa y Docencia en Red. Editorial Universitat Politècnica de València, págs. 1224-1234.

Stewart, Robert W y col. (2015). "A low-cost desktop software defined radio design environment using MATLAB, simulink, and the RTL-SDR". En: IEEE Communications Magazine 53.9, págs. 64-71.

Sánchez-Carracedo, F. y col. (2020). "La universidad que viene: de la "docencia remota de emergencia" a la "presencialidad adaptada"". En: The Conversation. 